\title{
Patogenia y tratamiento de la mucositis asociada al tratamiento de radioterapia y/o quimioterapia en pacientes con cáncer de cabeza y cuello.
}

Mathias Jeldres ORCID: 0000-0002-6600-9433 Residente tercer año de Oncología Clínica. Magister en ciencias médicas.

Dahiana Amarillo ORCID: 0000-0002-8615-8639

Oncóloga. Asistente del Departamento de Oncología Clínica. Asistente del Departamento Básico de Medicina.

Federico Lorenzo ORCID: 0000-0002-8929-7685 Oncólogo. Radioterapeuta. Servicio de Radioterapia Instituto Nacional del Cáncer. ASSE.

Federico Garcia ORCID: 0000-0002-5425-6234 Profesor Adjunto Medicina Interna.

Mauricio Cuello

ORCID: 0000-0003-1273-8080 Oncólogo. Profesor Agregado del Departamento de Oncología Clínica. Jefe policlínica oncología torácica y onco-otorrinolaringología.

\section{Pathogenesis and treatment of mucositis associated with radiotherapy} and / or chemotherapy treatment in patients with head and neck cancer.

Patogênese e tratamento da mucosite associada ao tratamento com radioterapia e / ou quimioterapia em pacientes com câncer de cabeça e pescoço.

Resumen: La mucositis es un efecto adverso frecuente e invalidante en los pacientes oncológicos que reciben tratamiento de Radioterapia y Quimioterapia a altas dosis y muchas veces lleva a la suspensión del tratamiento. Si bien es una entidad que tiene gran relevancia en los pacientes e importante impacto económico en las instituciones de salud, no existen tratamientos claramente establecidos ni eficaces para mejorar esta condición. El objetivo de esta revisión es analizar la evidencia disponible en el tratamiento de la mucositis, y el respaldo científico e impacto que tienen conductas habitualmente tomadas en su tratamiento.

Palabras clave: mucositis oral- dolor bucal- quimioterapia- tratamientos del cancer- radioterapia orl.

Abstract: Mucositis is a frequent and disabling adverse effect in cancer patients who received radiation therapy and chemotherapy at high doses and often discontinues treatment. Although it is an entity that has great relevance in patients and an important economic impact in health institutions, there are no clearly established or modified treatments to improve this condition. The objective of this review is to analyze the available evidence in the treatment of mucositis, and the scientific support and impact of behaviors commonly taken in its treatment.

Key words: oral mucositis- mouth pain- Chemotherapy- Cancer treatment- oral radiation.

Resumo: A mucosite é um efeito adverso frequente e incapacitante em pacientes com câncer que receberam radioterapia e quimioterapia em altas doses e muitas vezes interrompe o tratamento. Embora seja uma entidade que tenha grande relevância nos pacientes e um importante impacto econômico nas instituições de saúde, não existem tratamentos claramente estabelecidos ou modificados para melhorar essa condição. O objetivo desta revisão é analisar as evidências disponíveis no tratamento da mucosite, o suporte científico e o impacto das condutas comumente adotadas no seu tratamento.

Palavras-chave: mucosite oral, dor oral, quimioterapia, tratamentos contra 0 câncer, radioterapia oral

Recibido: 12/09/2020 Aceptado: 15/02/2021

Servicio de Oncología Clínica. Hospital de Clínicas. Facultad de Medicina. Universidad de la República. Montevideo, Uruguay.

Correspondencia. E-mail: mathiasjeldres@gmail.com 


\section{Introducción}

La mucositis es un efecto adverso frecuente secundario al tratamiento en pacientes oncológicos. Se refiere al daño (inflamación y ulceración) de la mucosa de la cavidad oral y del resto del tracto gastrointestinal y puede ser causado por la quimioterapia (QT) o la radioterapia (RT).

La incidencia de mucositis en el paciente oncológico depende del tratamiento recibido y la localización del tumor primario. Se estima que del 20 al $40 \%$ de los pacientes que reciben tratamiento sistémico con QT pueden presentar mucositis. Por otro lado, puede verse hasta en el $80 \%$ de los que reciben altas dosis de QT previo al trasplante para neoplasias hematológicas y en casi todos los pacientes que reciben tratamiento combinado de RT y QT en los cánceres de cabeza y cuello (1-2).

Los trabajos que sustentan el uso de RT combinada con QT en base a cisplatino en los cánceres de cabeza y cuello informan cifras de mucositis de entre el $40 \%$ al $80 \%$, y aproximadamente un $40 \%$ son grado 3 o 4. Según datos de la Organización Mundial de la Salud (OMS), los pacientes que reciben RT en tumores de cabeza y cuello tienen una incidencia de mucositis oral grado 3 o 4 cercana al $85 \%(2-3)$.

De todas maneras, la incidencia ha disminuido en los últimos años con el advenimiento de técnicas de RT moderna como la intensidad modulada, reportándose en un ensayo randomizado una disminución de 52 a $25 \%$ para carcinomas de cavidad oral. ${ }^{(4)}$.

Su importancia está dada por el impacto que a menudo genera en la calidad de vida. La mucositis severa puede requerir nutrición parenteral, genera dolor, complicaciones infecciosas e interrumpe el tratamiento pautado, y a diferencia de otros efectos adversos es de difícil manejo y no existen tratamientos claramente establecidos. Es importante tener en cuenta, además, que la interrupción de los tratamientos en pacientes con tumores de cabeza y cuello empeora la tasa respuesta y la sobrevida ${ }^{(5)}$.

Adicionalmente, se ha demostrado que la interrupción de la RT determina $1.2 \%$ de pérdida de control local por día de retraso en el tratamiento. ${ }^{(6)}$.

El objetivo de esta revisión es analizar la evidencia disponible en el tratamiento de la mucositis, y el respaldo científico e impacto que tienen conductas habitualmente tomadas en su tratamiento. Nuestra intención es ofrecer recomendaciones actualizadas para la práctica y manejo de pacientes con tumores de cabeza y cuello en tratamiento con radio-quimioterapia.

\section{Material y métodos}

Se analizó en primera instancia la evidencia disponible en las principales guías de manejo clínico de la mucositis utilizadas en oncología.

Guía "MASCC/ISOO clinical practice guidelines for the management of mucositis secondary to cancer therapy", publicada en Octubre $2020{ }^{(1)}$.

Guía ESMO 2015 "Management of oral and gastrointestinal mucosal injury"publicada en $2015^{(7)}$.

Posteriormente se realizó una búsqueda ordenada, en el buscador Pubmed, de la evidencia de cada tratamiento propuesto para la mucositis, utilizando como palabras clave combinaciones que incluyen el tratamiento propuesto, la afección a tratar -/+ la localización del cáncer. (ejemplo Benzydamine and mucositis and head and neck cancer). 2020.

Se limitó la búsqueda por tiempo, tomando publicaciones desde el año 2000 a diciembre

Finalmente se realizó una búsqueda siguiendo los mismos lineamientos de tratamientos originales, no mencionados en las guías de práctica clínica.

\section{Resultados}

\section{A) Patogenia}

La mucositis se produce en un complejo conjunto de pasos biológicos que afectan, no solo el epitelio, sino también a la submucosa. Se plantea una serie de 5 etapas en el proceso de 
mucositis: iniciación, respuesta al daño primario, amplificación de señal, ulceración, y curación (5).

Estas etapas constan de una serie de eventos biológicos que comienzan con el daño del ADN producidos por la RT o QT, y la producción de especies reactivas del oxígeno (ROS), que desencadenan los pasos posteriores. Se genera por estas vías la activación de NF-kB, que puede modular la transcripción de genes como los de las citocinas proinflamatorias IL-1B e IL-6 y TNF alfa, que promueven metaloproteinasas que destruyen la mucosa y la submucosa ${ }^{(5)}$.

La destrucción de las mucosas favorece la colonización por microorganismos y patógenos bacterianos, que potencian el proceso inflamatorio.

Hong et al. en 2019 analizaron el rol de la disbiosis del microbioma oral producido por la quimioterapia (específicamente 5-FU y adriamicina) en los primeros ciclos. Los resultados experimentales muestran que la gravedad de la mucositis podría estar relacionado con el enriquecimiento de ciertas especies bacterianas de la cavidad oral, por ejemplo, especies gram negativas y la depleción de especies vinculadas a la salud bucal. No se observaron cambios importantes en la población fúngica. El mismo estudio evidenció que la severidad de la mucositis debida al tratamiento con 5-FU ocurrió con un aumento del flujo de saliva y si bien los neutrófilos sanguíneos son depletados con el tratamiento, los mismos siguen siendo reclutados en la cavidad oral, lo que promovería los efectos deletéreos ${ }^{(8)}$.

Otro factor importante que considerar en la perpetuación de la mucositis son las coinfecciones, que pueden deberse o no a los tratamientos administrados. La coinfección que más frecuentemente se da en la mucositis producida durante el tratamiento oncológico con quimioterapia y radioterapia es la candidiasis. Se ha demostrado la reactivación viral del virus herpes simple debido al tratamiento de quimioterapia, generando mucositis en un gran porcentaje de pacientes ${ }^{(9)}$.

\section{B) Diagnóstico y estadificación}

El diagnóstico de mucositis oral es clínico, se basa en una correcta anamnesis y examen físico, junto con la relación temporal entre el comienzo del tratamiento y los síntomas y signos de mucositis ${ }^{(9)}$.

Existen varias escalas para clasificarla. Unas de las más utilizadas en las guías europeas y americanas es la de la OMS (figura 1):

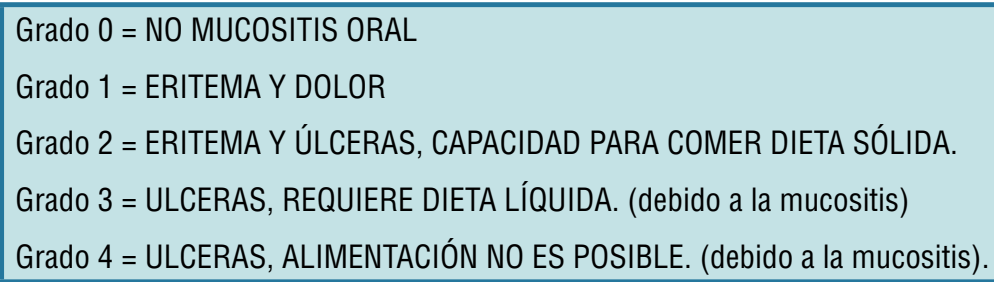

\section{C) Prevención y tratamiento de la mucositis en pacientes con tratamiento para tumores de cabeza y cuello}

Se han realizado a nivel de la comunidad médica y científica múltiples esfuerzos para entender la fisiopatología de la mucositis y así encontrar intervenciones que reduzcan su gravedad, aunque por el momento no contamos con una medida considerada estándar.

En el año 2019 fue actualizada una revisión sistemática del tratamiento de la mucositis oral realizada por la MASCC (Multinational Association of Supportive Care in Cancer), con versiones previas publicadas en el año 2013, 2007 y 2004.

Las siguientes Recomendaciones para profesionales de la salud que asisten a este grupo de pacientes se enumeran en las guías ESMO (Annals of Oncology, ESMO Guidelines) (9) Tabla 2. 
Tabla 2: Recomendaciones terapéuticas para tratamiento de la mucositis. Tomado de

RECOMENDACIONES

NO FARMACOLOGICAS:

-Inspección de mucosa oral.

-Cepillado de dientes y encías con cepillo suave.

-Enjuagues bucales sin alcohol.

-Evitar estímulos dolorosos, principalmente alcohol y tabaco.

-Cuidado de prótesis dentales.

\section{FARMACOLÓGICAS:}

Láser de baja potencia, previo a cada sesión de RT de lunes a viernes durante el tratamiento. (nivel de evidencia moderado-alto). (Disponible en Uruguay).

Enjuagues bucales mágicos, en Uruguay: Gelal, Xylocaína, Daktarin gel ${ }^{\circledR}$ (nivel de evidencia bajomoderado).

Bencidamina en pacientes con RT a dosis moderada. (<50 Gy). (nivel de evidencia moderado).

Bencidamina en pacientes que reciben RT a dosis $>50$ Gy (nivel de evidencia bajo-moderado).

Tea tree oil (nivel de evidencia bajo).

Crioterapia (nivel de evidencia bajo).

Diclofenac enjuague bucal (nivel de evidencia bajo).

\section{C.1) No farmacológicas}

Con respecto a los cuidados orales básicos, existe evidencia inadecuada y contradictoria y al igual que en el 2013 no hay directrices con respecto a la indicación de un cuidado bucal profesional. De todas maneras, la recomendación de expertos respalda esta medida y recomienda el tratamiento previo al comienzo del tratamiento oncológico. ${ }^{(10-11)}$.

Algunos ejemplos de medidas de cuidados orales básicos son numerados en guías internacionales, los cuales prevendrían la mucositis severa: mantenimiento de un soporte nutricional óptimo durante todo el período de tratamiento y estimular una rutina diaria de higiene bucal.

Con estas medidas, se plantea que se puede contribuir a la reducción y prevención de la lesión del tejido oral y el dolor asociado a la misma, el compromiso nutricional que genera y el resto de los resultados adversos relacionados.

Medidas generales. Inspeccione la mucosa oral en cada visita médica. Indique valoración por odontólogo previo al comienzo del tratamiento a modo de eliminar potenciales agentes que incrementen el daño (por ejemplo, prótesis mal ajustadas, dientes fracturados, focos sépticos). En caso de extracciones dentarias, deben mediar al menos diez días con el inicio del tratamiento. Recomiende lubricación de los labios con vaselina (estéril) / parafina blanca (vaselina), bálsamo labial o crema para labios durante el tratamiento. Debe insistirse en ingesta de una gran cantidad de líquidos para mantener la boca húmeda.

Cepillado de dientes y encías. Recomiende un cepillo de dientes suave o un hisopo (según lo tolerado) después de las comidas y antes de dormir. Esto reduce el riesgo de sangrado. De usarse cepillo de dientes suave, el mismo debe cambiarse de forma mensual. Debe utilizarse para dentadura y encía una pasta de dientes suave que no contenga fluoruro. El cepillo debe enjuagarse bien con agua después del y guardado en una taza con la cabeza hacia arriba. Si el paciente acostumbra la limpieza inter-dental, que consulte a un higienista dental/dentista sobre limpiador apropiado (hilo dental, palillo de dientes, cepillos). En caso de que no esté acostumbrado a hacerlo de forma regular, que no comience mientras esté en tratamiento, ya que puede romper la barrera epitelial visible a través del sangrado gingival.

Enjuague la boca. Enjuague la boca con un enjuague bucal sin alcohol al despertar y al menos cuatro veces al día después del cepillado, durante $1 \mathrm{~min}$, realizando gárgaras y escupiendo el sobrante. Durante la primera media hora después del enjuague, evite comer y beber. De no tener apertura bucal adecuada, utilice jeringas de mediano calibre (ej 20-60cc) para introducir el líquido de forma suave, tratando de alcanzar toda la cavidad bucal.

Cuidado de la dentadura postiza. Que el paciente retire las dentaduras postizas antes de realizar el cuidado bucal y las cepille con pasta dental y enjuague con agua. Debemos indicar que 
limpie sus encías como se mencionó previamente. Se debe aplazar el uso de prótesis dentales tanto como sea posible hasta que los tejidos del revestimiento de la boca estén curados.

Evitar estímulos dolorosos. Insistir en que se abandone el cigarrillo y la ingesta de alcohol. Además, es importante aconsejar que se eviten ciertos alimentos como tomates, frutas cítricas, bebidas calientes (té, mate, café) y comidas picantes, calientes, crudas o crujientes.

\section{C.2) Farmacológicas}

Existen escasas recomendaciones farmacológicas para el tratamiento y prevención de la mucositis generada por RT y QT en pacientes con tumores de cabeza y cuello. Algunas de ellas presentan una mayor evidencia científica, aunque siempre la indicación debe ser cuidadosa. Va a ser muy importante el manejo analgésico, pudiendo requerir en ocasiones tratamiento sistémico siguiendo la escalera de la OMS.

Palifermina. La palifermina (Kepivance $®$ ), es un factor de crecimiento queratinocítico (KGF) humano, producido mediante tecnología de ADN recombinante en Escherichia coli. Es el único agente aprobado por la FDA y la EMA para el tratamiento de la mucositis oral, particularmente el vinculado a pacientes adultos con neoplasias hematológicas sometidos a tratamiento de radioquimioterapia mielosupresora ${ }^{(12)}$.

La evidencia de su utilización data de un artículo publicado en el año 2004 por Spielberger ${ }^{(13)}$. Dicho estudio comparó la eficacia de la palifermina vs placebo, demostrando una reducción en la incidencia de mucositis oral grado 3 o 4, la mediana de duración de la mucositis y la disminución del dolor y el uso de nutrición parenteral con el tratamiento con palifermina en dicho grupo de pacientes. ${ }^{(13)}$. Al momento de realizar esta revisión, no se encuentra disponible en nuestro país.

La palifermina no tiene evidencia en caso de tumores sólidos y por lo tanto no se recomienda su utilización en neoplasias no hematológicas.

Bencidamina. La Bencidamina es un antinflamatorio y analgésico tópico, utilizado en enjuagues para el tratamiento de la mucositis y otras afecciones de la cavidad oral.

Las pautas internacionales analizadas recomiendan el enjuague bucal con bencidamina para prevenir la mucositis oral en pacientes con cáncer de cabeza y cuello que reciben RT de dosis moderada (hasta 50 Gy), sin tratamiento concomitante de QT ${ }^{(7)}$, en base a los resultados de un ensayo randomizado doble ciego entre otros ${ }^{(14)}$.

Sin embargo, es importante aclarar que estos resultados no pudieron reproducirse en un ensayo fase III posterior, que se detuvo en el primer análisis interino por futilidad y nunca fue publicado ${ }^{(15)}$.

En 2018 la Agencia Canadiense de drogas y tecnología en Salud realizó un reporte que revisó la evidencia y pautas para esta recomendación concluyendo la falta de evidencia que respalde estrategias de tratamiento seguras y efectivas para la mucositis, y no respalda la recomendación de la Bencidamina en el tratamiento de esta ${ }^{(16)}$

En cuanto al tratamiento de la mucositis vinculada a RT a dosis mayores a 50 Gy, un estudio aleatorizado, doble ciego que comparó la Bencidamina profiláctica vs placebo (iguales características sin el producto activo), concluyó que en el grupo de tratamiento hubo una diferencia estadísticamente significativa en la mucositis de grado $>=3$ en los dos grupos, que fue del $43,6 \%(n=17)$ en el grupo de bencidamina y del $78,6 \%(n=33)$ en el grupo de placebo $(P=0,001)$. Además, la mucositis de grado $>=3$ fue 2,6 veces más frecuente en el grupo de placebo $(R R=2,6$, IC del $95 \%=1,38-5)$. Sin embargo restan trabajos con un mayor número de pacientes y seguimiento para reforzar esta indicación. ${ }^{(17)}$

Otro estudio aleatorizado, no enmascarado, reunió 120 pacientes y los dividió primero acorde al tratamiento recibido en RT-QT concurrente o RT sola. Y estos grupos fueron aleatorizados a recibir Bencidamina $0.15 \%$ o placebo. Los resultados mostraron una reducción significativa en las tasas de mucositis de grado 3 en pacientes tratados con dosis de radiación $>50$ Gy (62.1 vs $35.4 \% ; p=0,038$ ) y menor, aunque no una reducción estadísticamente significativa en las tasas de mucositis de grado 3 o superior en pacientes tratados con QT concurrente (64.3 vs $43.3 \%$; $\mathrm{p}=0,091){ }^{(18)}$

Láser de baja potencia (LLLT). Ensayos clínicos prospectivos fase III, demostraron que la terapia con LLLT puede ser útil en la prevención de mucositis oral en pacientes sometidos a trasplante de células madre hematopoyéticas ${ }^{(19)}$. 
En los tumores de cabeza y cuello, contamos con varios ensayos randomizados que han evaluado el beneficio de esta estrategia.

Uno de ellos, estudio de Schubert et al, evaluó la eficacia de LLLT para reducir la incidencia de mucositis en los pacientes sometidos a QT - RT concurrente y su impacto en la calidad de vida. Este estudio fase III, doble ciego, aleatorizado, incluyó 94 pacientes con cánceres de cabeza y cuello locorregionalmente avanzados y los randomizó en 2 grupos que recibieron LLLT preventivo o placebo.

Los pacientes recibían un total de 70,2 Gy concurrente con cisplatino 100mg/ m2 cada 3 semanas, con criterio radical. Todos los pacientes recibían higiene bucal con cepillo especial, pasta dental, enjuague bucal con $0,12 \%$ de clorhexidina dos veces al día durante la RT. El LLLT preventivo se aplicó diariamente, durante 5 días consecutivos (de lunes a viernes), todas las semanas, inmediatamente antes de cada fracción de RT.

En este estudio se detectó una disminución significativa de la incidencia de mucositis grado 3 y 4 (RR 0.158 -IC 95\% 0.050-0.498). En cuanto a la calidad de vida se demostraron mejoras en el funcionamiento emocional, fatiga, deglución y dolor a favor de LLLT vs placebo ${ }^{(20)}$. El seguimiento a largo plazo de este estudio en el año 2017 publicó resultados de análisis de sobrevida. Los pacientes sometidos a LLLT mostraron un aumento en la sobrevida libre de progresión comparados con el grupo placebo (61.7\% vs. 40.4\%; $p=0.030$; HR: 1: 93; IC 95\%: $1.07-3.5)$ y una tendencia a mejor sobrevida global $(57.4 \%$ vs. $40.4 \%$; $p=0.90$; HR: 1.64 ; IC 95\%: 0.92-2.91). ${ }^{(21)}$

En el año 2019 se publicó otro estudio fase III que comparaba el uso de LLLT con placebo, que demostró resultados similares en cuanto a la disminución de la mucositis, pero los resultados no fueron significativos y el estudio carece de poder estadístico ${ }^{(22)}$.

Crioterapia. El uso de crioterapia es recomendado únicamente en pacientes que reciben QT con 5-FU en bolo, donde se ha demostrado que prevendría la aparición y severidad de la mucositis. Se indica 30 min previos a la utilización de este. ${ }^{(23)}$.

"Enjuagues bucales mágicos". Los denominados en inglés "Magic mouthwash", son una serie de preparaciones de medicamentos mixtos utilizados para prevenir o tratar la mucositis oral. Las formulaciones no son fijas y varían dependiendo de cada país e instituciones.

Habitualmente consisten en agentes anticolinérgicos (ej: difenhidramina), antiácidos y / o agentes protectores de la mucosa (ej: hidróxido de aluminio), anestésicos (ej: lidocaína) y, a veces, antibacterianos, antifúngicos, opioides y esteroides. En nuestro país la preparación más utilizada conjuga gelal $₫$ (hidróxido de aluminio), xylocaina (anestésico local) y daktarin gel $\AA$ (nistatina como antifúngico).

Una pequeña revisión de la literatura publicada en el 2018 muestra que no existe evidencia que apoye el uso de enjuagues bucales de medicamentos mixtos en el tratamiento de la mucositis inducida por QT. Se resalta la heterogeneidad de las formulaciones y la no presencia de esta recomendación en las pautas internacionales para el t ratamiento de la mucositis ${ }^{(24)}$. Su uso tampoco se recomienda en revisiones previas. ${ }^{(1)}$.

Sin embargo, una respuesta a esta publicación aclara, que si bien no existe evidencia que respalde el uso de estos preparados en la mucositis asociada a la quimioterapia, si la hay para la producida por la RT. Esto se basa en un estudio fase III publicado a modo de abstract, aleatorizado, doble ciego, controlado con placebo, que demostró que la preparación con estos 3 agentes disminuyó significativamente el dolor vs placebo.

Aunque dicha formulación no incluía nistatina, sino que se basaba en un antihistamínico, antiácido y lidocaína. ${ }^{(25)}$.

Tea tree oil. Es un aceite volátil esencial, que deriva de la planta nativa australiana Malaleuca Alternifolia. Se ha incorporado en el tratamiento de varias afecciones y es un agente de investigación actual en patologías cutáneas y oftalmológicas ${ }^{(25)}$.

Su composición presenta aproximadamente 100 sustancias distintas, principalmente monoterpenos, sesquiterpenos y alcoholes relacionados. Existen diferentes quimiotipos de plantas y condiciones de almacenamiento, pero a pesar de su variabilidad, la composición para su comercialización está regulada por una norma internacional, que especifica la concentración que deben cumplir 14 componentes del aceite. ${ }^{(26)}$. 
Se plantea que el principal componente con actividad biológica, particularmente antimicrobiana, es el terpinen-4-ol, el cual debe formar parte de la formulación del producto con un límite inferior de $30 \%$. Sin embargo, un artículo del 2018 publicado por Brun et al, sugiere que dicho componente podría no ser el principal agente antibacteriano del compuesto, atribuyendo su eficacia al sinergismo de estos ${ }^{(27)}$.

En Uruguay es ampliamente utilizado, ya que se cuenta con una presentación farmacéutica en el mercado, como tratamiento de soporte para pacientes oncológicos que reciben tratamiento de RT y QT.

El tea tree oil tiene actividad antimicrobiana, antifúngica y antiviral. La actividad antimicrobiana fue demostrada tanto in vitro como in vivo, y posee actividad bactericida y bacteriostática de acuerdo con las diferentes concentraciones ${ }^{(28)}$. Según ensayos in vitro, componentes del tea tree oil permeabilizan la membrana bacteriana para facilitar la penetración de otros componentes dirigidos a elementos importantes de la cadena respiratoria, y alteran genes que regulan la formación de biofilms y estructuras adhesivas. ${ }^{(28-30)}$.

El tea tree oil es activo contra una gran variedad de bacterias, como Streptococcus, Staphylococcus, Pseudomona aeruginosa, Lactobacillus, Klebsiella Pneumoniae, entre otros. (26)

La actividad antifúngica del tea tree oil fue limitada en su comienzo a Candida Albicans, que es el modelo de estudio más utilizado, pero existe evidencia de su eficacia en distintas levaduras, dermatofitos y otros hongos filamentosos. Su acción antifúngica no difiere mucho de la antibacteriana, alterando principalmente la membrana y su funcionamiento ${ }^{(27-30)}$.

En cuanto a la actividad anti viral, no hay tantos estudios publicados, pero se ha demostrado que tiene efecto sobre HSV-1 y 2 probablemente directo y sobre el virus libre, disminuyendo la formación de placa cuando el virus es tratado con el tea tree oil previamente a la absorción (26).

Además de sus efectos antimicrobianos, un estudio del año 2019 analizó el rol del tea tree oil in vitro en diferentes líneas celulares de cáncer de piel (A-375 melanoma y HEp-2 carcinoma de células escamosas). Los mismos mostraron que tiene la capacidad de generar muerte celular por apoptosis, a través de una vía dependiente de P53 ${ }^{(31)}$. Cabe destacar que esto no ha sido comprobado en ensayos clínicos.

Morfina. Un estudio con 30 pacientes con cáncer de cabeza y cuello con mucositis grave (grado III o IV de la Organización Mundial de la Salud) los asignó de forma aleatoria a recibir sulfato de morfina al 2\% o "Magic mouthwash" (que contenía hidróxido de aluminio y magnesio, lidocaína viscosa y difenhidramina), $10 \mathrm{ml}$ cada $3 \mathrm{~h}$, seis veces al día, durante 6 días. Ambos grupos recibieron las mismas instrucciones y cuidados de higiene bucal y dietética. Se observó una diferencia al sexto día de tratamiento con mayor reducción en la gravedad de la mucositis en el grupo de morfina en comparación con el grupo mágico $(P=0,045)$. Sin embargo se requieren estudios con mayor número de pacientes y seguimiento para confirmar estos resultados ${ }^{(32)}$.

Diclofenac. Un estudio de observación clínica evaluó en 10 pacientes sometidos a QT y RT de cáncer de cabeza y cuello, el valor potencial del enjuague bucal con diclofenac $(0,74 \mathrm{mg} / \mathrm{ml})$ para reducir los síntomas. El estudio mostró reducción del dolor en 9/10 pacientes, sin embargo este resultado debe ser evaluado en estudios randomizados ${ }^{(33)}$.

\section{D) Tratamiento y/o prevención de las complicaciones infecciosas}

Candidiasis. La candidiasis es la sobreinfección más frecuente en pacientes que tienen mucositis debida al tratamiento onco-específico. Aun así no existe consenso en el tratamiento profiláctico.

El 95\% es causada por Cándida Albicans, si bien existen más de 150 especies de Candida descriptas. El tratamiento de la candidiasis puede ser tópico o sistémico.

Habitualmente la candida oral se maneja con tratamiento tópico, requiriendo tratamiento sistémico únicamente si es una enfermedad recurrente o no responde al tratamiento local. Existen variantes de tratamiento tópico entre ellas miconazol tableta bucal de $50 \mathrm{mg}$ una vez al día durante 7-14 días o suspensión de nistatina (100,000 U / mL, 4-6 ml, cuatro veces al día). En nuestro país se utiliza frecuentemente el miconazol en gel a dosis según prospecto de 2,5 $\mathrm{ml}$ de gel 4 veces por día. En cuanto al tratamiento sistémico es recomendado en infecciones moderadas a severas, y a dosis de 100-200 mg al día durante 7-14 días de fluconazol oral. (34). 
Herpes simple. La coinfección con herpes simple tipo 1 es frecuente en pacientes que tienen mucositis debida al tratamiento oncológico. Habitualmente no es considerada como se debiera, pero la infección por herpes simple hace que la mucositis sea más grave y prolongada. El tratamiento en pacientes inmunocompetentes es a dosis habituales 200 a 400 mg por vía oral de dos a cinco veces al día por 7-10 días con aciclovir o $1 \mathrm{gr} /$ dìa por 7 dias para el valaciclovir ${ }^{(35)}$.

\section{Conclusiones}

La mucositis es una importante afección vinculada al tratamiento de radioterapia y quimioterapia en pacientes oncológicos, principalmente en cánceres de cabeza y cuello.

Es frecuentemente limitante de dosis y causa de interrupción del tratamiento, genera dolor, necesidad de nutrición parenteral y gran afectación de la calidad de vida. Existe escasa evidencia que respalde la utilización de ciertos productos contra la mucositis, que queda claro en las recomendaciones de las diferentes guías internacionales. Es fundamental además tomar los cuidados y medidas tópicas adecuadas en vistas a intentar su prevención y minimizar la morbilidad que genera.

Realizamos en esta revisión un análisis de la información disponible acerca del tratamiento de la mucositis, intentando ofrecer recomendaciones actualizadas. Creemos que, en base a lo expuesto, la prevención y tratamiento de esta afección se basa en un estricto control del cuidado de la mucosa oral y evitando los principales agentes nocivos como el alcohol y el tabaco.

En cuanto al tratamiento estándar utilizado en Uruguay, como enjuague bucal mágico no existe evidencia sólida de su utilización como mezcla, por lo que el tratamiento de cada complicación con sus componentes individuales es la opción más recomendada.

De estar disponible la utilización del láser de baja potencia previo a cada sesión de RT, puede ser recomendada.

La bencidamina parece ser el tratamiento con más evidencia disponible, sin embargo, la misma es contradictoria. Podría recomendarse como tratamiento profiláctico en pacientes que reciben $\mathrm{RT}$ a dosis moderada, sin evidencia para pacientes que reciben tratamiento concurrente de RT-QT.

El tratamiento de las complicaciones infecciosas debe realizarse con su tratamiento estándar.

Como perspectivas, sería de interés la comparación de los agentes disponibles en estudios randomizados prospectivos. Nuestro equipo se plantea la realización de un estudio prospectivo que compare un agente utilizado frecuentemente en nuestro medio como es el tea tree oil vs tratamientos estándar.

\section{Conflicto de interés}

Los autores declaran no tener conflicto de interés en el siguiente artículo de revisión.

\section{Bibliografía}

1- Elad S, Cheng KKF, Lalla RV, Yarom N, Hong C, Logan RM, et al. MASCC/ISOO clinical practice guidelines for the management of mucositis secondary to cancer therapy. Cancer. 2020 Oct 1;126(19):4423-4431. doi: 10.1002/cncr.33100.

2- Vera-Llonch M, Oster G, Hagiwara M, Sonis S. Oral mucositis in patients undergoing radiation treatment for head and neck carcinoma. Cancer. 2006 Jan 15;106(2):329-36. doi: 10.1002/cncr.21622.

3- Forastiere AA, Goepfert H, Maor M, Pajak TF, Weber R, Morrison W, et al. Concurrent chemotherapy and radiotherapy for organ preservation in advanced laryngeal cancer. N Engl J Med. 2003; 349(22):2091-8. doi: 10.1056/NEJMoa031317.

4- Wang ZH, Zhang SZ, Zhang ZY, Zhang CP, Hu HS, Tu WY, et al. Protecting the oral mucosa in patients with oral tongue squamous cell carcinoma treated postoperatively with intensity-modulated radiotherapy: a randomized study. Laryngoscope. 2012 Feb;122(2):291-8. doi: 10.1002/lary.22434.

5- Kwon Y. Mechanism-based management for mucositis: option for treating side effects without compromising the efficacy of cancer therapy. Onco Targets Ther. 2016 Apr 5;9:2007-16. doi: 10.2147/ OTT.S96899. 
6- González Ferreira JA, Jaén Olasolo J, Azinovic I, Jeremic B. Effect of radiotherapy delay in overall treatment time on local control and survival in head and neck cancer: Review of the literature. Rep Pract Oncol Radiother. 2015 Sep-Oct;20(5):328-39. doi: 10.1016/j.rpor.2015.05.010.

7- Peterson DE, Boers-Doets CB, Bensadoun RJ, Herrstedt J; ESMO Guidelines Committee. Management of oral and gastrointestinal mucosal injury: ESMO Clinical Practice Guidelines for diagnosis, treatment, and follow-up. Ann Oncol. 2015 Sep;26 Suppl 5:v139-51. doi: 10.1093/annonc/ mdv202.

8- Hong BY, Sobue T, Choquette L, Dupuy AK, Thompson A, Burleson JA, et al. Chemotherapy-induced oral mucositis is associated with detrimental bacterial dysbiosis. Microbiome. 2019;7(1):66. doi: 10.1186/s40168-019-0679-5.

9- Hong J, Park HK, Park S, Lee A, Lee YH, Shin DY, et al. Strong association between herpes simplex virus- 1 and chemotherapy-induced oral mucositis in patients with hematologic malignancies. Korean $J$ Intern Med. 2020 Sep;35(5):1188-1198. doi: 10.3904/kjim.2018.469.

10- Hong CHL, Gueiros LA, Fulton JS, Cheng KKF, Kandwal A, Galiti D, et al. Systematic review of basic oral care for the management of oral mucositis in cancer patients and clinical practice guidelines. Support Care Cancer. 2019 Oct;27(10):3949-3967. doi: 10.1007/s00520-019-04848-4.

11- McGuire DB, Fulton JS, Park J, Brown CG, Correa ME, Eilers J, et al. Systematic review of basic oral care for the management of oral mucositis in cancer patients. Support Care Cancer. 2013 Nov;21(11):3165-77. doi: 10.1007/s00520-013-1942-0.

12- National Cancer Institute. FDA approval for palifermin [Internet]. NCl; 2015 [acceso: 12/12/2020]. Disponible en: https://www.cancer.gov/about-cancer/treatment/drugs/palifermin

13- R. Spielberger ,P. Stiff, W.Bensinger, T. Gentile, et al. "Palifermin for oral mucositis after intensive therapy for hematologic cancers." N Engl J Med 2004 Dec 16;351(25):2590-8.

14- J Epstein JB, Silverman S Jr, Paggiarino DA, Crockett S, Schubert MM, Senzer NN, et al. Benzydamine $\mathrm{HCl}$ for prophylaxis of radiation-induced oral mucositis: results from a multicenter, randomized, doubleblind, placebo-controlled clinical trial. Cancer. 2001 Aug 15;92(4):875-85.

15- McNeil Consumer \& Specialty Pharmaceuticals. Safety \& efficacy study of benzydamine oral rinse for the treatment of oral mucositis (mouth sores) resulting from radia- tion therapy for cancer of the oral cavity, oropharynx, or nasopharynx [Internet]. NIH; 2011[access: 17/01/2020] NCT00051441. Available in: https://clinicaltrials.gov/ct2/show/NCT00051441?id=NCT00051441\&draw=2\&rank=1\&load=cart

16- MacDonald E, Visintini S. Benzydamine for the Treatment of Oropharyngeal Mucositis from Radiation Therapy: A Review of Clinical Effectiveness and Guidelines. Canadian Agency for Drugs and Technologies in Health: Ottawa; 2018.

17- Kazemian A, Kamian S, Aghili M, Hashemi FA, Haddad P. Benzydamine for prophylaxis of radiationinduced oral mucositis in head and neck cancers: a double-blind placebo-controlled randomized clinical trial. Eur J Cancer Care (Engl). 2009 Mar;18(2):174-8. doi: 10.1111/j.1365-2354.2008.00943.x

18- Rastogi M, Khurana R, Revannasiddaiah S, Jaiswal I, Nanda SS, Gupta P, et al. Role of benzydamine hydrochloride in the prevention of oral mucositis in head and neck cancer patients treated with radiotherapy (>50 Gy) with or without chemotherapy. Support Care Cancer. 2017 May;25(5):1439-1443. doi: 10.1007/s00520-016-3548-9.

19- Bourbonne V, Otz J, Bensadoun RJ, Dissaux G, Lucia F, Leclere JC, et al. Radiotherapy mucositis in head and neck cancer: prevention by low-energy surface laser. BMJ Support Palliat Care. 2019 Sep 16:bmjspcare-2019-001851. doi: 10.1136/bmjspcare-2019-001851.

20- Schubert MM, Eduardo FP, Guthrie KA, Franquin JC, Bensadoun RJ, Migliorati CA, et al. A phase III randomized double-blind placebo-controlled clinical trial to determine the efficacy of low level laser therapy for the prevention of oral mucositis in patients undergoing hematopoietic cell transplantation. Support Care Cancer. 2007 Oct;15(10):1145-54. doi: 10.1007/s00520-007-0238-7

21- Antunes HS, Herchenhorn D, Small IA, Araújo CMM, Viégas CMP, et al. Long-term survival of a randomized phase III trial of head and neck cancer patients receiving concurrent chemoradiation therapy with or without low-level laser therapy (LLLT) to prevent oral mucositis. Oral Oncol. 2017 Aug;71:11-15. doi: 10.1016/j.oraloncology.2017.05.018.

22- Legouté F, Bensadoun RJ, Seegers V, Pointreau Y, Caron D, Lang P, et al. Low-level laser therapy in treatment of chemoradiotherapy-induced mucositis in head and neck cancer: results of a randomised, triple blind, multicentre phase III trial. Radiat Oncol. 2019 May 22;14(1):83. doi: 10.1186/s13014-019$1292-2$

23- Peterson DE, Ohrn K, Bowen J, Fliedner M, Lees J, Loprinzi C, et al. Systematic review of oral cryotherapy for management of oral mucositis caused by cancer therapy. Support Care Cancer. 2013 Jan;21(1):327-32. doi: 10.1007/s00520-012-1562-0. 
24- Uberoi AS, Brown TJ, Gupta A. Magic Mouthwash for Oral Mucositis: A Teachable Moment. JAMA Intern Med. 2019 Jan 1;179(1):104-105. doi: 10.1001/jamainternmed.2018.6223.

25- Miller RC, Le-Rademacher J, Sio TTW, Leenstra JL, Rine GP, Curtis A. A Phase III, Randomized Double-Blind Study of Doxepin Rinse versus Magic Mouthwash versus Placebo in the Treatment of Acute Oral Mucositis Pain in Patients Receiving Head and Neck Radiotherapy with or without Chemotherapy (Alliance A221304). Radiation Oncology. Red Journal. 2013; 85(1): 21.

26- Carson CF, Hammer KA, Riley TV. Melaleuca alternifolia (Tea Tree) oil: a review of antimicrobial and other medicinal properties. Clin Microbiol Rev. 2006 Jan;19(1):50-62.

27- International Organisation for Standardisation. (AS 2019)2004. ISO 4730 (HS 2017):2004. Oil of Melaleuca, terpinen-4-ol type (tea tree oil). ISO: Geneva; 2019.

28- Brun P, Bernabè G, Filippini R, Piovan A. In Vitro Antimicrobial Activities of Commercially Available Tea Tree (Melaleuca alternifolia) Essential Oils. Curr Microbiol. 2019 Jan;76(1):108-116. doi: 10.1007/ s00284-018-1594-x.

29- Ramage G, Milligan S, Lappin DF, Sherry L, Sweeney P, Williams C, et al. Antifungal, cytotoxic, and immunomodulatory properties of tea tree oil and its derivative components: potential role in management of oral candidosis in cancer patients. Front Microbiol. 2012 Jun 18;3:220. doi: 10.3389/ fmicb.2012.00220

30- Gustafson JE, Liew YC, Chew S, Markham J, Bell HC, Wyllie SG, Warmington JR. Effects of tea tree oil on Escherichia coli. Lett Appl Microbiol. 1998 Mar;26(3):194-8. doi: 10.1046/j.1472-765x.1998.00317.x.

31- Ramadan MA, Shawkey AE, Rabeh MA, Abdellatif AO. Expression of P53, BAX, and BCL-2 in human malignant melanoma and squamous cell carcinoma cells after tea tree oil treatment in vitro. Cytotechnology. 2019 Feb;71(1):461-473. doi: 10.1007/s10616-018-0287-4.

32- 32- Sarvizadeh M, Hemati S, Meidani M, Ashouri M, Roayaei M, Shahsanai A. Morphine mouthwash for the management of oral mucositis in patients with head and neck cancer. Adv Biomed Res. 2015 Feb 11;4:44. doi: 10.4103/2277-9175.151254.

33- Brennan PA, Lewthwaite R, Sakthithasan P, McGuigan S, Donnelly O, Alam P Brennan PA, Lewthwaite R, Sakthithasan P, McGuigan S, Donnelly O, Alam P, et al. Diclofenac Mouthwash as a potential therapy for reducing pain and discomfort in chemo-radiotherapy-induced oral mucositis. J Oral Pathol Med. 2020 Oct;49(9):956-959. doi: 10.1111/jop.13001.

34- Quindós G, Gil-Alonso S, Marcos-Arias C, Sevillano E, Mateo E, Jauregizar N, et al. Therapeutic tools for oral candidiasis: Current and new antifungal drugs. Med Oral Patol Oral Cir Bucal. 2019 Mar 1;24(2):e172-e180. doi: 10.4317/medoral.22978.

35- Tyring SK, Baker D, Snowden W. Valacyclovir for herpes simplex virus infection: long-term safety and sustained efficacy after 20 years' experience with acyclovir. J Infect Dis. 2002 Oct 15;186 Suppl 1:S406. doi: $10.1086 / 342966$.

\section{Aporte de cada autor al trabajo}

Mathias Jeldres: concepción y diseño del trabajo, recolección de datos, redacción y revisión crítica del manuscrito.

Dahiana Amarillo: concepción y diseño del trabajo, recolección de datos, redacción y revisión crítica del manuscrito.

Federico Lorenzo: concepción y diseño del trabajo, revisión crítica del manuscrito.

Federico Garcia: concepción y diseño del trabajo, revisión crítica del manuscrito.

Mauricio Cuello: concepción y diseño del trabajo, revisión crítica del manuscrito. 